\title{
The Resummation of One Particle Lines ${ }^{\star}$
}

\author{
James Glimm ${ }^{1}$ and Arthur Jaffe ${ }^{2}$ \\ 1 The Rockefeller University, New York, New York 10021, USA \\ ${ }^{2}$ Harvard University, Cambridge, Massachusetts 02138, USA
}

\begin{abstract}
We propose a partial resummation for a weak coupling cluster expansion. The resummation gives one particle lines with in/out field propagators. We give a Bethe-Salpeter equation in which one particle subtractions are defined using physical one particle states. By these methods, we show that $P(\phi)_{2}$ quantum fields in the weak coupling region have only isolated bound state spectrum below the $2 m$ threshold. Here $P$ is not restricted to be even.
\end{abstract}

\section{Introduction}

We present a new method for the study of mass spectrum, asymptotic completeness and related questions. The method uses exact subtractions of the physical one particle states; technically, it is based on the $n$-particle cluster expansion [8], and a (new)partial resummation which identifies the physical one particle lines in closed form.

Previous work $[3,4,11,12]$ on spectral properties of $P(\phi)_{2}$ quantum fields was based on the Euclidean program of defining $n$-particle irreducible amplitudes [13]. In this program, the vacuum subtractions are performed exactly, while one and higher particle subtractions are replaced by an orthogonalization of the polynomials in the Euclidean field $\phi$. Thus in this program, a $j$-particle irreducible $n$ point function is defined by projecting $\phi\left(x_{1}\right) \phi\left(x_{2}\right) \ldots \phi\left(x_{n}\right)$ onto the orthogonal complement, in the Euclidean Hilbert space $\mathscr{E} \equiv L_{2}\left(\mathscr{S}^{\prime}, d \mu\right)$, of the subspace spanned by the vectors

$$
\phi\left(y_{1}\right) \ldots \phi\left(y_{l}\right), l \leqq j, y_{v} \in \mathbb{R}^{2} .
$$

While the resulting subtraction agrees with the subtraction of (physical) $j$-particle intermediate states for $j=0$, it does not agree in general, and in particular if bound

* Supported in part by the National Science Foundation under Grants PHY 78-08066 and PHY 7718762. Both authors thank the I.H.E.S., Bures-sur-Yvette, and A. J. thanks C.E.N., Saclay, for their hospitality. 
states occur. For $j \geqq 1$ spurious zeros and poles (CDD poles) can be introduced by these subtractions.

The Euclidean program has been successful when $j=0$ (the vacuum) was the only subtraction required. Within this limit, particles and bound states below the continuum threshold have been analyzed, and for even theories asymptotic completeness up to energies below the four particle threshold is known. Various extensions of this work have been given $[1,2,7,9]$. This work makes it appears likely that the $j$ particle Euclidean subtractions can be incorporated into the spectral analysis program.

We study here the simplest problem requiring a one particle subtraction: the analysis of the four point function up to energies below the three particle threshold, for a general (not necessarily even) interaction at weak coupling. These methods appear useful in the study of higher spectral properties in field theory, and presumably they have an analog in statistical mechanics.

In $\S 2$, we define normal ordering: $\phi\left(x_{1}, 0\right) \phi\left(x_{2}, 0\right)$ : of the square of the time $t=0$ field by subtraction of the physical one particle state. In other words, if $|\Omega\rangle$ is the vacuum state in the physical Hilbert space $\mathscr{H}$, then

$$
\left.: \phi^{2}:|\Omega\rangle \perp \mid \text { one particle states }\right\rangle .
$$

The central object of the study in this paper is then

$$
S_{0, T}=\left\langle\Omega\left|: \phi\left(x_{1}, 0\right) \phi\left(x_{2}, 0\right):: \phi\left(y_{1}, T\right) \phi\left(y_{2}, T\right):\right| \Omega\right\rangle,
$$

where $\Omega \equiv 1$ is the Euclidean vacuum state, and the inner product is in the Euclidean Hilbert space $\mathscr{E}$. We let $\langle\cdot\rangle$ now denote an expectation in the Euclidean vacuum $\Omega$. Let

$$
\begin{aligned}
G_{0, T}= & \left\langle\phi\left(x_{1}, 0\right) \phi\left(y_{1}, T\right)\right\rangle\left\langle\phi\left(x_{2}, 0\right) \phi\left(y_{2}, T\right)\right\rangle \\
& +\left\langle\phi\left(x_{1}, 0\right) \phi\left(y_{2}, T\right)\right\rangle\left\langle\phi\left(x_{2}, 0\right) \phi\left(y_{1}, T\right) .\right.
\end{aligned}
$$

We replace the Euclidean Bethe - Salpeter equation of the form (in momentum space)

$$
S^{\sim}=G^{\sim}+G^{\sim} K^{\sim} S^{\sim},
$$

by an equation of the form

$$
S_{0, T}=A_{0, T}+\sum_{0 \leqq j \leqq T} B_{0, T} S_{j, T} .
$$

By summing over $T$, this leads to an equation

$$
\sum_{0 \leqq T} S_{0, T}=\sum_{0 \leqq T} A_{0, T}+\left(\sum_{0 \leqq j} B_{0, j}\right) \sum_{0 \leqq T} S_{0, T}
$$

with the solution

$$
\sum_{0 \leqq T} S_{0, T}=\left(1-\sum_{0 \leqq j} B_{0, j}\right)^{-1} \sum_{0 \leqq T} A_{0, T},
$$

since $S_{0, T}=S_{j, T+j}$, etc. We see that $\sum_{0 \leqq j} B_{0, j}$ plays the role of $G^{\sim} K^{\sim}$ in the BetheSalpeter equation. As with $G^{\sim} K^{\sim}$, we achieve a factorization of $\sum_{0 \leqq j} B_{0, j}$. The first 
factor contains the free field behavior of the four point function, and has a square root singularity with a two-sheeted analytic continuation. The first factor arises from two physical one particle lines, up to the time of their first interaction. The second ("amputated") factor is analytic up to energies less than $3(m-\varepsilon)$, and contains the effects of interaction between the one particle lines. Moreover $\sum_{0 \leqq j} B_{0, j}$ is compact on a suitable space of functions, analytic in $p$. The spectral properties of the field theory, for $E \leqq 3(m-\varepsilon)$, follow from these facts.

\section{One Particle Subtractions}

In this section we review properties of one particle subtractions and establish notation. To simplify the formulas, we assume

$$
\langle\Omega|\phi(x, t)| \Omega\rangle=0 .
$$

In other words, for a general theory, we make the substitution $\phi \rightarrow \hat{\phi} \equiv \phi-\langle\phi\rangle$. Let $|p\rangle$ be the one particle state of momentum $p$. with the normalization

$$
\left\langle p \mid p^{\prime}\right\rangle=(2 \mu(p))^{-1} \delta\left(p-p^{\prime}\right),
$$

where $\mu(p)=\left(p^{2}+m^{2}\right)$ and $m$ is the one particle mass. Then with

$$
\begin{aligned}
& |x\rangle=(2 \pi)^{-1 / 2} \int e^{-i p x}|p\rangle d p \\
& |f\rangle=\int f(x)|x\rangle d x,
\end{aligned}
$$

we have

$$
\langle x \mid y\rangle=(2 \mu)^{-1}(x-y) ;\langle f \mid g\rangle=\left\langle f,(2 \mu)^{-1} g\right\rangle_{L_{2}},
$$

where $\mu=\left(-d^{2} / d x^{2}+m^{2}\right)^{1 / 2}$. The field strength renormalization constant $Z$ is defined by

$$
\begin{aligned}
& \left\langle y\left|Z^{-1 / 2} \phi(x, 0)\right| \Omega\right\rangle=(2 \mu)^{-1}(x-y), \quad \text { or equivalently } \\
& Z^{1 / 2}=(2 \pi)^{1 / 2} e^{i p x} 2 \mu(p)\langle p|\phi(x)| \Omega\rangle .
\end{aligned}
$$

By Lorentz invariance, $Z$ is independent of $x, y, p$.

Let $M_{0}=|\Omega\rangle\langle\Omega|$ and let

$$
M_{1}-M_{0}=\int|p\rangle\langle p| 2 \mu(p) d p
$$

be the orthogonal projection onto one particle states. In general, $M_{a}$ is the projection onto states of mass $M \equiv\left(H^{2}-P^{2}\right)^{1 / 2} \leqq a m$. Then $M_{0} \phi \Omega=0$,

$$
M_{1} \phi(f, 0)|\Omega\rangle=Z^{1 / 2}|f\rangle,
$$

and

$$
\begin{aligned}
e^{-t H} M_{1} \phi(f, 0)|\Omega\rangle & =Z^{1 / 2} e^{-t H}|f\rangle \\
& =Z^{1 / 2}\left|e^{-t \mu} f\right\rangle=M_{1} \phi\left(e^{-t \mu} f, 0\right)|\Omega\rangle .
\end{aligned}
$$


In weakly coupled $P(\phi)_{2}$ theories, as considered in this paper, $I-M_{1}$ projects onto states with energy greater than $2\left(m_{0}-\varepsilon\right)$, where $m_{0}$ is the bare mass. Thus

$$
\begin{aligned}
& \| e^{-T H}\left\{e^{-H} \phi(f, 0)|\Omega\rangle-\phi\left(e^{-\mu} f, 0\right)|\Omega\rangle\right\} \| \\
& \quad \leqq 0(1) e^{-2\left(m_{0}-\varepsilon\right) T} .
\end{aligned}
$$

We use this formula in $\S 5$ to identify resummed one particle lines.

We define the (physical) normal ordering, so that $: \phi^{2}:|\Omega\rangle \perp M_{1}$. Let

$$
\begin{aligned}
& \alpha_{0}(x-y)=\langle\Omega|\phi(x, 0) \phi(y, 0)| \Omega\rangle \\
& \alpha_{1}(z-x, x-y)=Z^{-1 / 2}\left(2 \mu_{z}\right)\langle z|\phi(x, 0) \phi(y, 0)| \Omega\rangle .
\end{aligned}
$$

Then

$$
\begin{aligned}
: \phi(x, 0) \phi(y, 0):= & \phi(x, 0) \phi(y, 0)-\alpha_{0}(x-y) \\
& -\int \alpha_{1}(z-x, x-y) \phi(z, 0) d z .
\end{aligned}
$$

We require for later use regularity properties of $\alpha_{0}$ and $\alpha_{1}$. Note $\alpha_{0}$, as a truncated Schwinger function, is $C^{\infty}$ except at $x-y=0$, where it has a $\ln$ singularity, and $\alpha_{0}$ and all derivatives are exponentially decreasing as $x-y \rightarrow \infty$. The estimates on $\alpha_{1}$ in this section are elementary. They will be improved in $\S 5$. We work mainly in a space of fixed total momentum $p$, and so we seek a bound on

$$
\begin{aligned}
\tilde{\alpha}_{1}(p, x-y) & \equiv(2 \pi)^{-1 / 2} \int e^{+i p(x+y-2 z) / 2} \alpha_{1}(z-x, x-y) d z \\
& =Z^{-1 / 2} 2 \mu(p)\left\langle p\left|\phi\left(\frac{x-y}{2}, 0\right) \phi\left(\frac{y-x}{2}, 0\right)\right| \Omega\right\rangle .
\end{aligned}
$$

Let $\mathscr{H}(p)$ denote the Hilbert space of fixed total momentum $p$. with inner product $\langle,\rangle_{p}$. It is convenient to exclude $\Omega$ from $\mathscr{H}(0)$, so that

$$
\mathscr{H} \oplus\{\lambda \Omega\}=\int_{\oplus} \mathscr{H}(p) d p .
$$

In general, vectors in $\mathscr{H}(p)$ are continuum states in $\mathscr{H}$. We can, however, given $\Phi$, $\Psi \in \mathscr{H}-\{\lambda \Omega\}$ write a formula for $\langle\Phi(p), \Psi(p)\rangle_{p}$, namely

$$
\langle\Phi(p), \Psi(p)\rangle_{p}=\langle\Phi, \Psi\rangle_{p}=(2 \pi)^{-1 / 2} \int\left\langle\Phi, e^{-i p a} T_{a} \Psi\right\rangle_{\mathscr{H}} d a,
$$

where $T_{a} \equiv e^{i P a}$ is translation by $a$.

From the exponential cluster properties of the truncated Schwinger functions, we see that

$$
\left\{\phi(x, 0) \phi(y, 0)-\alpha_{0}(x-y)\right\} \Omega
$$

has finite norm in $\mathscr{H}(p)$, with a bound uniform in $x, y$. Optimal estimates give

$$
\int f(x-y)\left\{\phi(x, 0) \phi(y, 0)-\alpha_{0}(x-y)\right\} \Omega d(x-y)=\theta
$$

a finite norm in $\mathscr{H}(p)$ when $f$ is in the Sobolev space $H_{-1}$, and in fact

$$
\|\theta(p)\|_{p}^{2} \leqq \text { const. }\left\|\mu^{-1} f\right\|_{L_{2}} \text {. }
$$

Such bounds are true more generally of vectors

$$
\Psi=\left[\phi\left(x_{1}, t_{1}\right) \ldots \phi\left(x_{n}, t_{n}\right)-\left\langle\phi\left(x_{1}, t_{1}\right) \ldots \phi\left(x_{n}, t_{n}\right)\right\rangle\right] \Omega,
$$


with all $t_{j} \geqq 0$ and no two $\left(x_{j}, t_{j}\right)$ 's coinciding. Such estimates are proved by integration by parts, cf. [5], to obtain optimal momentum space behavior (i.e. free field singularities at coinciding points in $x$ space). Such vectors $\Psi$ (when smoothed with test functions to become elements of $\mathscr{H}$ ) are dense in $\mathscr{H}$. Thus the corresponding vectors (2.20) are dense in $\mathscr{H}(p)$ for a.e. $p$. Furthermore they are continuous in $p$ in the sense that $\left\langle\psi_{1}(p), \psi_{2}(p)\right\rangle_{p}$ is continuous in $p$.

With $f \in \mathscr{S}$, let

$$
\theta_{t}=\phi\left(e^{t \mu} f, t\right) \Omega=\int \phi(x, t)\left(e^{t \mu} f\right)(x) d x \Omega .
$$

A calculation with the Lehmann spectral formula shows that as $t \rightarrow \infty$, the vectors $\theta_{t}$ converge in $\mathscr{H}$ at the rate $O\left(e^{-t(m-\varepsilon)}\right)$. The same is true, furthermore for $\theta_{t}(p)$ in $\mathscr{H}(p)$, with convergence uniform in $p$. The limit is

$$
\lim _{t \rightarrow \infty} \theta_{t}=Z^{1 / 2} \Phi_{e x}(f) \Omega,
$$

where the time zero asymptotic field $\Phi_{e x}$ is given by standard Haag-Ruelle scattering theory. We now use $\Phi$ to denote the real time (Minkowski) field, and $\Phi_{e x}$ its asymptotic limit. At time zero we identify the Euclidean and Minkowski fields $\Phi(x) \equiv \Phi(x, 0)=\phi(x, 0) \equiv \phi(x)$.

We claim that after mapping $\Phi_{\text {ex }}(f) \Omega$ into $\mathscr{H}\left(p_{0}\right)$ by (2.16), we may take the limit $f(p) \rightarrow \delta\left(p-p_{0}\right)$ in the test function. Thus we identify $\tilde{\Phi}_{e x}(p) \Omega \tilde{f}(p)$ as the component of $\Phi_{e x}(f) \Omega$ with momentum $p$.

To justify this exchange of limits first appeal to the uniformity of the convergence in (2.22) as $t \rightarrow \infty$ in each $\mathscr{H}(p)$. It follows that the inner product $\left\langle\Psi, \Phi_{e x}(f) \Omega\right\rangle_{p}$ is a continuous function of $p$. Take $\tilde{f}(p)=\tilde{f}_{1}(p) \tilde{f}_{2}(p)$. By (2.16)

$$
\left\langle\Psi, \Phi_{e x}(f) \Omega\right\rangle_{p}=\left\langle\Psi, \Phi_{e x}\left(f_{1}\right) \Omega\right\rangle_{p} \tilde{f}_{2}(p) .
$$

Because of the continuity of the inner product in $p$, we can take the limit $\tilde{f}_{2}(p) \rightarrow \delta\left(p-p_{0}\right)$, where $\tilde{f}_{1}\left(p_{0}\right)=1$.

Thus

$$
\left\langle\Psi, \tilde{\Phi}_{e x}\left(p_{0}\right) \Omega\right\rangle_{\mathscr{H}}=\int\left\langle\Psi, \Phi_{e x}(f) \Omega\right\rangle_{p} d p
$$

and we define $\tilde{\Phi}_{e x}\left(p_{0}\right) \Omega$ as an improper element of $\mathscr{H}$. The vector $\tilde{\Phi}_{e x}(p) \Omega$ has a finite inner product with all vectors $\Psi$ of the form (2.20). Thus also by (2.23),

$$
\left\langle\Psi, \tilde{\Phi}_{e x}\left(p_{0}\right) \Omega\right\rangle_{p}=\left\langle\Psi, \tilde{\Phi}_{e x}(p) \Omega\right\rangle_{\mathscr{H}} \delta\left(p-p_{0}\right) .
$$

Since the definition, $\left|p_{0}\right\rangle=\tilde{\Phi}_{e x}\left(p_{0}\right) \Omega$, it follows that $\left\langle\Psi \mid p_{0}\right\rangle$ is also well defined and bounded uniformly in $\left(x_{j}, t_{j}\right)$ for all $t_{j} \geqq 0$ and no coinciding points, unless $n=2$. In particular, since $\left\langle p_{0} \mid \Omega\right\rangle=0$, it follows that $\tilde{\alpha}_{1}(p, x-y)$ is bounded as a function of $x-y$. Furthermore, $\tilde{\alpha}_{1}(p, x-y)$ is Hölder continuous in $x-y$ with exponent less than $1 / 2$.

In terms of the function spaces $A_{\delta}$ of $\S 6, \tilde{\alpha}_{1}(p, \cdot) \in A_{\delta}$ for $\delta_{1} \leqq 3 / 2-\varepsilon, \delta_{2}<0$. To obtain this improvement on the Hölder continuity above, we write

$$
\begin{aligned}
\left\langle p \mid \phi\left(x_{1}\right) \phi\left(x_{2}\right) \Omega\right\rangle & =e^{\mu(p)}\left\langle p \mid e^{-H} \phi\left(x_{1}\right) \phi\left(x_{2}\right) \Omega\right\rangle \\
& =e^{\mu(p)}\left\langle p \mid e^{-H}\left(I-M_{0}\right) \phi\left(x_{1}\right) \phi\left(x_{2}\right) \Omega\right\rangle,
\end{aligned}
$$


and the bound the norm of $e^{-H}\left(I-M_{0}\right) \Phi\left(x_{1}\right) \phi\left(x_{2}\right) \Omega$ in $\mathscr{H}(p)$. The improved smoothing comes from $e^{-H}$ and can be exhibited by integration by parts formulas as above. Then (2.19) is replaced by $\left\|\mu^{-3 / 2+\varepsilon} f\right\|_{L_{2}}$.

\section{Span of : $\Phi^{n}$ :}

Let

$$
: \Phi^{j}(f, t): \equiv e^{i t H}: \Phi\left(x_{1}\right) \ldots \Phi\left(x_{j}\right): e^{-i t H}
$$

where : $\Phi\left(x_{1}\right) \ldots \Phi\left(x_{j}\right):$ denotes normal ordering defined so that $: \Phi:=\Phi, M_{0} \Phi \Omega=0$, and

$$
M_{1}: \Phi\left(x_{1}\right) \ldots \Phi\left(x_{j}\right): \Omega=0, j \geqq 2 .
$$

Theorem 3.1. Choose $n$ integer $\geqq 2, \varepsilon>0$, and $\lambda<\lambda(n, \varepsilon)$. Then states of the form

$$
M_{n+1-\varepsilon}: \Phi^{j}(f, t): \Omega, \quad 2 \leqq j \leqq n,
$$

$\operatorname{span}\left(M_{n+1-\varepsilon}-M_{1}\right) \mathscr{H}$. Here $f$ ranges over $\mathscr{S}\left(\mathbb{R}^{n}\right)$, and $t$ belongs to an open interval $(a, b)$.

Remark. Taking $n=2$, the vectors $M_{3-\varepsilon}: \Phi^{2}:(f, t) \Omega$ span all bound states and two particle states with mass in the interval $(m,(3-\varepsilon) m)$. In other words, all states with mass in this interval occur in the four point function $S_{0, T}$.

Proof. Let $E_{a}$ denote the projection onto states with energy $\leqq a m$. Let $|f, t\rangle_{j} \equiv: \Phi^{j}:(f, t) \Omega$. We show that $E_{n+1-\varepsilon}|f, t\rangle_{j}, j=2, \ldots, n$, span $E_{n+1-\varepsilon}\left(I-M_{1}\right) \mathscr{H}$. By Lorentz invariance, the states $M_{n+1-\varepsilon}|f, t\rangle_{j}$ then span $\left(M_{n+1-\varepsilon}-M_{1}\right) \mathscr{H}$. For states $E_{a}|f, t\rangle_{j}$ we note that the total momentum $P$ satisfies

$$
P^{2} \leqq\left(a^{2}-1\right) m^{2}
$$

Secondly we remark that $E_{a}|f, t\rangle_{j}$ is an entire function of $t$. Thus if $\left\langle\chi\left|E_{a}\right| f, t\right\rangle_{j}=0$, for $t \in(a, b)$, it follows by analytic continuation that $\left\langle\chi\left|E_{a}\right| f, i t\right\rangle_{j}=0$. Thus it is sufficient to show that $E_{n+1-\varepsilon}|f, i t\rangle_{j}, j=2,3, \ldots, n, t \in \mathbb{Z}_{+}$ $\operatorname{span} E_{n+1-\varepsilon}\left(I-E_{1}\right) \mathscr{H}$.

It has already been proved by the cluster expansion [8] that the states

$$
E_{n+1-\varepsilon}\left\{\Omega, \Phi(f, t) \Omega,|f, i t\rangle_{j}, 2 \leqq j \leqq n, t \in \mathbb{Z}_{+}\right\},
$$

span $E_{n+1-\varepsilon} \mathscr{H}$. In fact the same method [8], taking (3.4) into account, shows that $\Phi(f, 0) \Omega$ can replace $\Phi(f, t) \Omega$ in (3.5). Thus a dense set of states $\chi$ in $E_{n+1-\varepsilon} \mathscr{H}$ have the form

$$
\chi=E_{n+1-\varepsilon}\{\alpha \Omega+\phi(f) \Omega+\psi\}
$$

where $\psi$ is in the span of $|f, i t\rangle, t \in \mathbb{Z}_{+}$and $2 \leqq j \leqq n$. In (3.6), we restrict $f$ to have Fourier transform $f^{\sim}(p)$ vanishing unless $p^{2} \leqq[(n+1-\varepsilon)-1] m^{2}$ c.f. (3.4).

We take a sequence $\chi_{n}$ of such vectors, converging to a $\chi \in\left(1-M_{1}\right) \mathscr{H}$. Then

$$
M_{0} \chi_{n}=\alpha_{n} \Omega \rightarrow M_{0} \chi=0
$$


so that $\alpha_{n} \rightarrow 0$. Since the norms $f \rightarrow\|\phi(f) \Omega\|$ and $f \rightarrow\left\|M_{1} \phi(f) \Omega\right\|$ are equivalent, and since

$$
\left(M_{1}-M_{0}\right) \chi_{n}=M_{1} \phi\left(f_{n}\right) \Omega \rightarrow M_{1} \chi=0 .
$$

$\phi\left(f_{n}\right) \Omega \rightarrow 0$ also. Thus $\psi_{n} \rightarrow \chi$ and the proof is complete.

\section{Integration by Parts}

The basic expansion steps depend on integration by parts. Let

$$
C_{0}=\left(-\Delta+M_{0}^{2}\right)^{-1}(x, y)=(2 \mu)^{-1}\left[\exp \left(-\left|x_{0}-y_{0}\right| \mu\right)\right](\mathbf{x}, \mathbf{y}),
$$

and let $C$ be a covariance of the class considered in [8]. This class is generated from $C_{0}$ by two operations: (a) the block diagonalization

$$
C \rightarrow \chi_{X} C \chi_{X}+\chi_{\mathbb{R}^{2} \backslash X} C \chi_{\mathbb{R}^{2} \backslash X}
$$

for $X$ a rectangle in $\mathbb{R}^{2}$, and (b) the convex sum

$$
\left\{C_{j}\right\} \rightarrow \sum \beta_{j} C_{j}, \sum \beta_{j}=1, \beta_{j} \geqq 0 .
$$

For this class of covariance operators, we define the normalized measure on $\mathscr{S}^{\prime}\left(\mathbb{R}^{2}\right)$,

$$
d \mu_{C}=\lim _{\Lambda \uparrow \mathbb{R}^{2}} Z(\Lambda)^{-1} \exp \left(-\int_{\Lambda} \mathscr{P}(\phi(x)) d x\right) d \mu_{C},
$$

with existence of the limit following from [8].

With $R(\phi)$ a polynomial in $\phi$, the formula

$$
\frac{D R(\phi)}{D \phi(y)}=\left[\frac{\partial}{\partial \phi(y)}-\mathscr{P}^{\prime}(\phi(y))\right] R(\phi)
$$

defines $D / D \phi(y)$ as a densely defined linear operator on $\mathscr{E}$. Integration by parts is the identity

$$
\phi(x)=\int_{R^{2}} C(x, y) \frac{D}{D \phi(y)} d y,
$$

valid when substituted in the integral

$$
\int \phi R(\phi) d \mu_{C} \text {. }
$$

We use (4.6) to decompose $\phi$ into a sum of "annihilation" and "creation" operators :

$$
\begin{aligned}
& \phi(x)=a(x)+a^{*}(x), \\
& a(x)=\int_{x_{0} \leqq y_{0}} C(x, y) \frac{D}{D \phi(y)} d y ; a(x)^{*}=\int_{x_{0} \geqq y_{0}} C(x, y) \frac{D}{D \phi(y)} d y .
\end{aligned}
$$

Next consider a time interval $(s, t)$ without contours. This means

$$
C(x, y)=C_{0}(x, y), s \leqq x_{0}, y_{0} \leqq t .
$$


Then for $x_{0} \leqq z_{0} \leqq y_{0}$

$$
C(x, y)=\int_{z_{0}=\text { const }} C_{0}(x, z)\left(2 \mu C_{0}\right)(z, y) d \mathbf{z},
$$

which has the consequence

$$
\begin{aligned}
\int_{x_{0} \leqq y_{0} \leqq z_{0}} C(x, y) \frac{D}{D \phi(y)} d y & =a(x)-\left(e^{-\left(z_{0}-x_{0}\right) \mu} a\right)\left(z_{0}, \mathbf{x}\right) \\
& \left.=a\left(z_{0}, \mathbf{x}\right)^{*}-\left(e^{-\left(z_{0}-x_{0}\right.}\right)^{\mu} a^{*}\right)(x) .
\end{aligned}
$$

These identities are used to write $\phi=a+a^{*}$ and to move $a, a^{*}$ forward and backward in time. Normally $a^{*}$ is moved to time $t=-\infty$, i.e. eliminated. Also $a$ $=\phi-a^{*}$ can be rewritten in terms of $\phi$.

Remaining expansion steps concern the insertion and removal of contours, achieved by variation of the parameters $\beta_{j}$ in (4.3).

\section{The One Particle Line}

The main technical tool of this paper is the use of virtual contraction terms to allow the resummation of one particle lines. We explain these ideas here, and for clarity we give a very simple example, namely the evaluation of

$$
\int \phi(f, 0) R_{T}(\phi) d \mu=\left\langle\phi(f, 0) R_{T}\right\rangle
$$

with $R_{T}$ localized at times $t \geqq T$. As an application, we obtain improved bounds on $\alpha_{1}$.

The goal of our expansion is to write

$$
\left\langle\phi(f, 0) R_{T}\right\rangle=\left\langle\{\alpha+\phi(w, 1)\} R_{T}\right\rangle+O\left(e^{-2\left(m_{0}-\varepsilon\right) T}\right),
$$

where the right hand side is a partial resummation of the $n$-particle cluster expansion given in [8]. Having identified the resummation leading to (5.2), we can use (5.2) as an elementary expansion step in the definition of our modified BetheSalpeter equation (1.6). In terms of vectors in the physical Hilbert space $\mathscr{H}$, we identify (5.2). Let

$$
|\chi\rangle \equiv \phi(f, 0)|\Omega\rangle-\langle\Omega|\phi(f, 0)| \Omega\rangle|\Omega\rangle-e^{H} \phi\left(e^{-\mu} f, 0\right)|\Omega\rangle .
$$

Then (2.10) says that for any $\theta=R_{0}|\Omega\rangle$,

$$
\left|\left\langle\chi, e^{-T H} \theta\right\rangle\right| \leqq O(1) e^{-2\left(m_{0}-\varepsilon\right) T},
$$

and comparison with (5.2) shows that $w=e^{-\mu} f, \alpha=\langle\phi(f)\rangle$.

In order to evaluate (5.1), we use the 1-particle expansion in [8]. Writing $\phi=a+a^{*}$, we use (4.8), (4.10) to advance the annihilator in time to become a field $\phi$ at $t=1$. We use (4.9) to contract $a^{*}$. New vertices from the $D / D \phi$ terms are removed by insertion and removal of contours, as in [8]. We divide the expansion terms into two sets as follows: Terms in the first set have $y_{0}<T$ (where $y_{0}$ is defined in [8, pp. 609-610]). These terms have the form $\langle Q R\rangle$, where

$$
Q=\sum_{j} Q_{j}, \quad Q_{j}=\alpha(j)+\phi\left(w(j), t_{j}\right),
$$


where $t_{j}$ is an integer time, $t_{j}<T$. The second set of terms has $y_{0}=+\infty$, and contains contradictions to times $\geqq T$. These are all remainder terms, and the sum of the norms of remainder terms is bounded by $O(1)\|\phi(f)\|\|R\| e^{-2\left(m_{0}-\varepsilon\right) T}$ with norms defined in [8]. Thus we write

$$
\langle\phi R\rangle=\langle Q R\rangle+\text { Remainder } .
$$

Furthermore, we choose $f(x)$ with $f^{\sim}$ supported in

$$
\{p:|p| \leqq \sqrt{3}(m-\varepsilon)\}=\left\{p: \mu(p) \leqq 2\left(m-\varepsilon^{\prime}\right)\right\} .
$$

Then the terms $\phi\left(w(j), t_{j}\right)$ in (5.5) can be returned to time $t=1$.

In summary, we have for $n=1$

$$
\langle\phi(f, 0) R\rangle=\alpha_{T}\langle R\rangle+\left\langle\phi\left(w_{T}, 1\right) R\right\rangle+O\left(e^{-2\left(m_{0}-\varepsilon\right) T}\right) .
$$

In (5.8), $\alpha_{T}$ and $\phi\left(w_{T}, 1\right)$ are the linear and constant terms in $Q$. Each term in $Q$ is independent of $T$. However writing $Q$ as a sum of terms $Q=\sum_{i} Q_{i}$, the index set $\{i\}$ depends on $T$. Thus $\alpha_{T}$ and $w_{T}$ also depend on $T$. In order to cure this defect, we add and subtract certain terms on the right side of (5.8). To identify the missing terms we consider the case $T=\infty$, when (5.8) becomes

$$
\langle\phi(f, 0) R\rangle=\alpha\langle R\rangle+\langle\phi(w, 1) R\rangle .
$$

For $T=\infty$, there are no error terms, but there are terms in $\alpha$ resulting from contraction of $\phi(f, 0)$ to the vacuum in the region $t \geqq T$. We can think of these terms as contractions to the vacuum in the region blocked by $R$, and we call these terms, virtual contractions of $\phi$ to $R$.

The sum of the virtual contraction terms is absolutely convergent and bounded by $O(\exp -2(m-\varepsilon) T)$, because this sum is contained in the expansion of (5.9) to which the estimates of [8] also apply. Thus

$$
\begin{aligned}
& \alpha p R\rangle-\alpha_{T}\langle R\rangle=\sum \text { virtual terms } \\
& \langle\phi(w, 1) R\rangle-\left\langle\phi\left(w_{T}\right) R\right\rangle=\sum \text { virtual terms }
\end{aligned}
$$

where in (5.10) we sum over all virtual terms which are independent of $\phi$, and in (5.11) the sums runs over all terms linear in $\phi$. Note that the estimates of [6] apply to operator kernels, such as $w_{T}, w_{T=\infty}$ and $w_{T=\infty}-w_{T}$, as well as to expectation values such as $\left\langle\phi\left(w_{T=\infty}, 1\right)\right\rangle$. In the norm of [8],

$$
\left\|w_{T=\infty}-w_{T}\right\| \leqq O\left(e^{-2(m-\varepsilon) T}\right) .
$$

We add and subtract (5.10) and (5.11) to the right side of (5.5). The addition modifies $\alpha$ and $w$, while the subtraction is absorbed into the error term $O\left(e^{-2\left(m_{0}-\varepsilon\right) T}\right)$. This yields

$$
\langle r(f, 0) R\rangle=\alpha\langle R\rangle+\langle\phi(w, 1) R\rangle+O\left(e^{-2(m-\varepsilon) T}\right),
$$

where now $\alpha=\alpha_{T-\infty}$ and $w=w_{T=\infty}$ are independent of $T$.

Now we identify $\alpha=\langle\phi(f, 0)\rangle$, and with the convention (2.1), $\alpha=0$. Comparison of (5.2) and (5.4) shows that $w=e^{-\mu} f$. 
Thus in (5.13), we have achieved an explicit resummation of the one particle line, with the exact physical mass. The error term is an absolutely convergent series of direct and virtual contraction terms, with two particle decay $\sim e^{-2(m-\varepsilon) T}$.

To conclude the description of the expansion, we correct an error in [8]. In order to return field operator $\phi(f, t)$ to earlier times $s<t$, we must be dealing with translation invariant, i.e., momentum conserving expansion operations. The expansion is based on an integer lattice, artificially introduced in position space. Because of the translation invariance of the original theory, the expansion is term by term invariant under integer (lattice preserving) translations, but not under arbitrary space translations. To recover full translation invariance, we integrate each term over relative lattice positions, generated by the translations

$$
\mathbb{Z} \rightarrow \mathbb{Z}+\theta, \theta \in(0,1]
$$

This integration is done at the end of each expansion step containing no contours, i.e. before moving $\phi(f, t)$ backward in time.

The norms of [8] are not invariant under translation by non-integer $\theta$, as in (5.14), but they are easily replaced by equivalent norms, which are $\theta$-invariant. This is done explicitly in $\S 6$. The expansion remains norm decreasing in these equivalent norms. The extra expansion step,

$$
F(\theta, \ldots) \rightarrow \int_{0}^{1} F(\theta, \ldots) d \theta
$$

is norm preserving, in the new translation invariant, norms. Thus the modified expansion is norm decreasing, and convergent, as before.

The improved estimates on $\tilde{\alpha}_{1}$ give exponential decrease, in addition to the local regularity established in $\S 2$. In terms of the space $A_{\delta}$ of $\S 6$, we state our result as

Theorem 5.1. As a function of $x_{\text {rel }}=(x-y) / 2, \tilde{\alpha}_{1}\left(p, x_{\text {rel }}\right) \in A_{\delta}$ for $\delta_{1}<2-\varepsilon, \delta_{2}>0$ sufficiently small. The norm of $\tilde{\alpha}_{1}$ goes to zero as $\lambda \rightarrow 0$.

We give part of the proof here, and complete the argument in $\S 9,10$. For $x_{\text {rel }}$ in a bounded set, the analysis of $\S 2$ is sufficient. Thus we let $j \leqq x_{\text {rel }} \leqq j+1$ and consider $j$ large. As in $\S 2$,

$$
\left\|\mid\left(\Phi_{e x}(f)-Z^{-1 / 2} e^{-j H}\left(\phi\left(e^{j \mu} f\right)\right)\right) \Omega>\right\| \leqq O\left(e^{-(m-\varepsilon) j}\right)
$$

by a calculation with the Lehmann spectral formula, and the same bound holds in each space $\mathscr{H}(p)$ of total momentum $p$. If $\chi_{j}$ represents a smoothed version of the characteristic function of $[j, j+1]$, then we see as in $\S 2$ that for $\chi_{j} \equiv \chi_{j}\left(x_{\text {rel }}\right)$,

$$
\chi_{j}\left\{\tilde{\alpha}_{1}\left(p_{\mathrm{tot}}, x_{\mathrm{rel}}\right)-\left\langle Z^{-1 / 2} e^{-j H} \phi\left(e^{j \mu} f\right) \Omega, \phi^{2}\left(x_{\mathrm{rel}}, p_{\mathrm{tot}}\right) \Omega\right\rangle_{p}\right\}
$$

has an $H_{3 / 2-\varepsilon}$ norm bounded by $e^{-(m-\varepsilon) j}$, and thus finite norm in $A_{\delta}$, which is moreover summable in $j$. By (5.2), we can write

$$
\begin{aligned}
\left\langle\phi\left(e^{l \mu} f, 0\right), \int \phi\left(x_{1}, l\right) \phi\left(x_{2}, l\right) g(x) d x\right\rangle \\
=\left\langle\phi\left(e^{(l-1) \mu} f, 0\right), \int \phi\left(x_{1}, l-1\right) \phi\left(x_{2}, l-1\right) g d x\right\rangle \\
\quad+O\left(e^{-2\left(m_{0}-\varepsilon\right) l}\right) e^{-\delta j}\left\|e^{l \mu} f\right\|\|g\|,
\end{aligned}
$$


for $1 \leqq l \leqq j$. The exponent proportional to $j$ will be explained below. The norms in the error term are from [8], and the error term can be bounded by $e^{-\varepsilon^{\prime} j}\|f\|\|g\|$, provided $p_{\text {tot }}$, i.e. the support of $f$, is restricted to $\left|p_{\text {tot }}\right| \leqq \sqrt{3} m_{0}\left(1-\varepsilon^{\prime \prime}\right)$. We now iterate this identity, and sum over $0 \leqq l \leqq j$ to obtain

$$
\begin{aligned}
& \left\langle\phi\left(e^{j \mu} f, 0\right), \int \phi\left(x_{1}, j\right) \phi\left(x_{2}, j\right) g(x) d x\right\rangle \\
& \quad=\left\langle\phi(f) \int \phi\left(x_{1}\right) \phi\left(x_{2}\right) g(x) d x\right\rangle+O\left(e^{-\varepsilon^{\prime} j}\right)\|f\|\|g\| .
\end{aligned}
$$

The first term has exponential decrease in $x_{\text {rel }}$. In fact because of the normalization $\langle\phi\rangle=0$, the ordinary and truncated three point functions coincide. Thus the first term, at constant momentum $p$, belongs to $A_{\delta}$ in the variable $x_{\text {rel }}$.

The error terms in (5.16) result from direct and virtual contraction to the observables $\phi\left(x_{1}, l-1\right)$ and $\phi\left(x_{2}, l-1\right)$. However terms with contraction to only one of these two observables can be resummed, and yield zero, again because of the normalization $\langle\phi\rangle=0$. The terms with direct or virtual contraction to both $\phi$ 's at time $l-1$ have extra decay, determined by $\mid x_{\text {rel }}$, i.e., $j$. The relation of the norms of [8], as in (5.17), to the norms in $A_{\delta}$ is analyzed in $\S 9,10$, and will complete the proof.

\section{Function Spaces}

In this section we define function spaces suitable for the analysis of our expansion. First we introduce spaces necessary to obtain a translation invariant expansion. Let $\left\{\Delta_{i}\right\},\left\{\Delta_{i}^{\prime}\right\}$ each be disjoint covers of $\mathbb{R}^{n}$, and define

$$
\begin{aligned}
\|f\| & =\sum_{i}\|f\|_{L_{2}\left(\Delta_{i}\right)} \\
\|f\|^{\prime} & =\sum_{i}\|f\|_{L_{2}\left(\Delta_{i}^{\prime}\right)} .
\end{aligned}
$$

We assume that each $\Delta_{i}$ is contained in the union of at most $K$ of the $\Delta_{i}^{\prime \prime}$ s, and conversely.

Proposition 6.1. The norms (6.1) and (6.2) are equivalent, and

$$
\frac{1}{K}\|f\|^{\prime} \leqq\|f\| \leqq K\|f\|^{\prime} .
$$

In addition, if $f_{a}(x)=f(x+a)$, then for all $a \in \mathbb{R}^{n}$, let

$$
\|f\|=\int_{\Delta}\left\|f_{a}\right\| d a,
$$

where $\Delta$ is a unit $n$-cube. We assume $\left(\Delta_{i}+a\right) \cap \Delta_{j} \neq \emptyset$ for at most $K j$ 's, for each fixed choice of $i$ and $a$.

Proposition 6.2. The norms (6.1) and (6.4) are equivalent.

As an application, we change norms in the expansion of [8]. In place of the norms (2.43) of [8], we replace $\left\|w\left(\alpha, u, s, y_{0}, \cdot\right)\right\|_{L_{2}}$ by

$$
\int_{0}^{1}\left\|w\left(\alpha, u, s, y_{0}, \cdot+a \theta\right)\right\|_{L_{2}} d \theta, \quad a=(1,1, \ldots, 1) .
$$


for a localized kernel $w$. As described in $\S 5$, the integration over $\theta$ arises in the expansion from integration over choices of the position of the space lattice. The step of averaging the expansion over $\theta$ preserves the sum of the (averaged) norms of elementary expansion terms.

A second application involves separation of center of mass coordinates. For a function $f\left(x_{1}, \ldots, x_{j}\right)$ of $j$ variables, we introduce

$$
\begin{aligned}
x_{\mathrm{tot}} & =\frac{1}{j} \sum_{k=1}^{j} x_{k} \\
x_{\mathrm{rel}, k} & =x_{k}-x_{\mathrm{tot}} .
\end{aligned}
$$

For $i \in \mathbb{Z}^{j}$ we define for $I_{k}=[k, k+1]$,

$$
\Delta_{i}=I_{i_{1}}\left(x_{\mathrm{rel}, i_{1}}\right) \ldots I_{i_{j-1}-1}\left(r_{\mathrm{rel}, j-1}\right) I_{i_{j}\left(x_{\mathrm{tot}}\right)} \text {. }
$$

With these new $\Delta$ 's in (6.7), we obtain a new set of equivalent norms. A translation invariant kernel $k(x ; y)$, acts on spaces of definite total momentum as follows:

$$
k_{\text {rel }}\left(x_{\text {rel }} ; y_{\text {rel }} ; p_{\text {tot }}\right)=(2 \pi)^{-1 / 2} \int e^{i p_{\text {tot }}\left(y_{\text {tot }}-x_{\text {tot }}\right)} k(x ; y) d y_{\text {tot }} .
$$

In the special case $j=2$, we deal with kernels for two particle scattering. These are kernels of integral operators from functions of a single relative variable $\frac{1}{2}\left(y_{1}-y_{2}\right)$ to functions of a single relative variable $\frac{1}{2}\left(x_{1}-x_{2}\right)$. Thus we introduce Hilbert spaces of functions of one variable on which these operators act. We use the space $A_{\delta}$ of symmetric functions with $\delta_{1}$ fractional $L_{2}$ derivatives and with Fourier transforms analytic in a strip $|\operatorname{Im} p|<\delta_{2} \leqq 4 m$, Our choice of the space $A_{\delta}$ is motivated by [12]. Related spaces are considered in [10].

We let $\delta=\left(\delta_{1}, \delta_{2}\right),\left|\delta_{2}\right|<4 \mathrm{~m}$, and let

$$
K_{\delta}=e^{\delta_{2}|x|} \mu^{\delta_{1}} \text {, }
$$

where $\mu=\left(-d^{2} / d x^{2}+(4 m)^{2}\right)^{1 / 2}$. Let us define the inner product

$$
\langle f, g\rangle_{\delta}=\left\langle K_{\delta} f, K_{\delta} g\right\rangle_{L_{2}} ;\|f\|_{\delta}=\left\|K_{\delta} f\right\| .
$$

Then $A_{\delta}$ is the Hilbert space $C_{0}^{\infty}$ completed in the norm $\|\cdot\|_{\delta}$. In $\S 7$ we show that $K_{\delta}^{*}$ may be used in place of $K_{\delta}$ in (6.10). Likewise, since

$$
K_{\delta}^{-1}=K_{-\delta}^{*},
$$

we can define $A_{\delta}$ as the unitary image under $K_{-\delta}^{*}$ of $L_{2}(R)$,

$$
K_{-\delta}^{*}: L_{2}(R) \rightarrow A_{\delta} .
$$

By (6.11), it follows that the dual space of $A_{\delta}$ is

$$
A_{\delta}^{*}=A_{-\delta} .
$$

We say that $\delta>\delta^{\prime}$ if $\delta>\delta_{1}^{\prime}$ and $\delta_{2}>\delta_{2}^{\prime}$. We state properties of $A_{\delta}$ in three propositions. 
Proposition 6.3. Let $\delta \geqq \delta^{\prime}$. Then

$$
A_{\delta} \subset A_{\delta^{\prime}}
$$

Let $I\left(\delta^{\prime}, \delta\right)$ denote the injection of $A_{\delta}$ into $A_{\delta^{\prime}}$, for $\delta \geqq \delta^{\prime}$,

$$
I\left(\delta^{\prime}, \delta\right): A_{\delta^{\prime}} \rightarrow A_{\delta^{\prime}}
$$

Proposition 6.4. Let $\delta>\delta^{\prime}$.

(i) $I\left(\delta^{\prime}, \delta\right)$ is compact.

(ii) If $\delta>\delta^{\prime}+\left(\frac{1}{2}, 0\right)$, then (6.15) is Hilbert Schmidt

(iii) If $\delta>\delta^{\prime}+(1,0)$, then (6.15) is trace class.

We let $A^{0}$ denote the Banach space of functions $f$ with norm

$$
\|f\|=\sum_{n}\|f\|_{L_{2}(n, n+1)}<\infty,
$$

Proposition 6.5. Let $\delta_{1} \geqq 0, \delta_{2}>0$. Then

$$
A_{\delta} \subset A^{0} \subset A_{-\delta} .
$$

Furthermore the injections $A_{\delta} \rightarrow A^{0} \rightarrow A_{-\delta}$ are bounded.

Proofs. In the proofs of these three propositions, we refer ahead to $\S 7$. The proof of Propositions 6.3-6.4 involve the study of the operator $K_{\delta^{\prime}} K_{\delta}^{-1}$. In fact let $\left\{f_{n}\right\}$ denote a sequence in $A_{\delta}$. Then

$$
K_{\delta^{\prime}} f_{n}=\left(K_{\delta^{\prime}} K_{\delta}^{-1}\right)\left(K_{\delta} f_{n}\right) \text {. }
$$

Thus if $\left\{f_{n}\right\}$ is bounded in $A_{\delta}$, i.e. $\left\|K_{\delta} f_{n}\right\|_{L_{2}} \leqq$ const., it is bounded (compact) in $A_{\delta^{\prime}}$ if $K_{\delta^{\prime}} K_{\delta}^{-1}$ is bounded (compact). By Theorem 7.6,

$$
K_{\delta^{\prime}} K_{\delta}^{-1}=e^{\delta_{2}^{\prime}|x|} \mu^{-\left(\delta_{1}-\delta_{1}^{\prime}\right)} e^{-\delta_{2}|x|}
$$

is bounded for $\delta \geqq \delta^{\prime}$ and compact for $\delta>\delta^{\prime}$. Furthermore, (6.19) is HilbertSchmidt under condition (ii) and trace class under condition (iii).

We now proceed to Proposition 6.5. Using Proposition 6.3,

$$
A_{\delta} \subset A_{0, \delta_{2}} \subset A_{0,-\delta_{2}} \subset A_{-\delta} .
$$

Thus it is sufficient to establish (6.17) for the case $\delta_{1}=0$. Let $\delta$ now denote $\delta_{2}>0$, and let

$$
M(\delta)=\sum_{n} e^{-\delta|n|}
$$

Then

$$
\|f\|_{-\delta}=\left\|e^{-\delta|x|} f\right\|_{L_{2}} \leqq M(\delta) \sup _{n}\|f\|_{L_{2}(n, n+1)} \leqq M(\delta)\|f\| .
$$

Also

$$
\|f\|_{L_{2}(n, n+1)} \leqq e^{-\delta(|n|-1)}\left\|e^{\delta|x|} f\right\|_{L_{2}(n, n+1)} .
$$


Thus by the Schwarz inequality

$$
\begin{aligned}
\|f\| & \leqq e^{\delta} M(2 \delta)^{1 / 2}\left(\sum_{n}\left\|e^{\delta|x|} f\right\|_{L_{2}(n, n+1)}^{2}\right)^{1 / 2} \\
& =e^{\delta} M(2 \delta)^{1 / 2}\|f\|_{\delta} .
\end{aligned}
$$

This completes the proof.

We end this section with a remark generalizing the consequences of (6.12) (6.18) for the injection $A_{\delta} \rightarrow A_{\delta^{\prime}}$.

Proposition 6.6. The transformation $R: A_{\delta} \rightarrow A_{\delta^{\prime}}$ is bounded, compact, Hilbert Schmidt or trace class if and only if the operator

$$
K_{\delta^{\prime}} R K_{\delta}^{-1}
$$

is bounded, compact, Hilbert Schmidt or trace class on $L_{2}(\mathbb{R})$.

\section{7. $L_{2}$ Estimates}

In this section let $\mu=\left(-d^{2} / d x^{2}+a^{2}\right)^{1 / 2}$, and let $k(\alpha, a ; x-y)$ denote the kernel of $\mu^{-\alpha}$. Note that $k(\alpha, a ; x)=a^{1-\alpha} k(\alpha, 1, a x)$, a scaling relation.

Proposition 7.1. Let $0<\alpha<1$. Then

$$
0<k(\alpha, a, x)<w(\alpha, a ; x) \equiv \text { const. }|x|^{-1+\alpha} e^{-a|x|} .
$$

If $\alpha>1$,

$$
0<k(\alpha, a, x)<w(\alpha, a ; x) \equiv \text { const. } a^{1-\alpha} e^{-a|x|} .
$$

Proof. Let $A$ be a strictly positive operator with bounded inverse. By the Cauchy integral formula, for $0<\alpha<2$,

$$
A^{-\alpha / 2}=\frac{\sin (\alpha \pi / 2)}{\pi} \int_{0}^{\infty}(A+b)^{-1} b^{-\alpha / 2} d b .
$$

Take $A=\mu^{2}$. The kernel of $(A+b)^{-1}$ is

$$
\begin{aligned}
(A+b)^{-1}(x, y) & =(2 \pi)^{-1 / 2} \int\left(p^{2}+a^{2}+b\right)^{-1} e^{-i p(x-y)} d p \\
& =(2 \pi)^{1 / 2}(2 m(b))^{-1} e^{-m(b)|x-y|},
\end{aligned}
$$

where $m(b)=\left(a^{2}+b\right)^{1 / 2}$. The positivity of $k$ then follows by the positivity of (7.3), (7.4). We now specialize to the case $a=1, \alpha<1$ from which (7.1) follows by scaling. Inserting (7.4) in (7.3) and dividing the $b$ integration into $\int_{0}^{1}+\int_{1}^{\infty}$, we first bound the $\int_{0}^{1}$ integral. For $0 \leqq b \leqq 1, m(b) \geqq 1+a / 4$. Thus

$$
\begin{aligned}
\int_{0}^{1}(A+b)^{-1}(x, 0) b^{-\alpha / 2} d b & \leqq \text { const. } e^{-|x|} \int_{0}^{1} e^{-b|x| / 4} b^{-\alpha / 2} d b \\
& \leqq \text { const. } e^{-|x|} \min \left\{|x|^{-1+\alpha / 2}, 1\right\} \\
& \leqq \text { const. }|x|^{-1+\alpha} e^{-|x|}
\end{aligned}
$$


For the $\int_{1}^{\infty}$ integral,

$$
\begin{aligned}
& \int_{1}^{\infty} m(b)^{-1} e^{-m(b)|x|} b^{-\alpha / 2} d b \\
& \quad=2 \int_{x}^{\infty} \frac{x}{\left(t^{2}+x^{2}\right)^{1 / 2}} t^{1-\alpha}|x|^{-2+\alpha} e^{-\left(t^{2}+x^{2}\right)^{1 / 2}} d t \\
& \quad \leqq 2|x|^{-1+\alpha} \int_{x}^{\infty} t^{-\alpha} e^{-\left(t^{2}+x^{2}\right)^{1 / 2}} d t .
\end{aligned}
$$

For $t \geqq x$, it follows that for $\varepsilon>0$ there exists $\beta<\infty$ satisfying

$$
\left(t^{2}+x^{2}\right)^{1 / 2} \geqq|x|+\varepsilon t-\beta .
$$

Thus (7.6) is bounded by

$$
\text { const }|x|^{-1+\alpha} e^{-|x|} \text {. }
$$

Combining (7.5), (7.7) yields (7.1).

If $\alpha>1$, we again obtain (7.2) by scaling the $m=1$ bound. In this case we use the $n$-fold convolution

$$
k(\alpha, 1, x)=w * \ldots * w, \quad w=k(\alpha / n, 1, x) \quad 0<\alpha / n<1,
$$

and the bound (7.1) to establish $k(\alpha, 1, x) \leqq$ const $e^{-|x|}$. Similarly

Proposition 7.2. Let $|x| \geqq \varepsilon>0,-1<\alpha<\infty$. Then

$$
\left|\left(\frac{d}{d x}\right)^{n} k(\alpha, a, x)\right| \leqq C_{n, \varepsilon, a} e^{-a|x|} .
$$

Theorem 7.3. Let $-1<\alpha<1,-a<\beta<a$. Then

$$
\mu^{\alpha} e^{\beta|x|} \mu^{-\alpha} e^{-\beta|x|} \text { and } e^{\beta|x|} \mu^{\alpha} e^{-\beta|x|} \mu^{-\alpha}
$$

are bounded, as operators on $L_{2}$

Proof. For simplicity consider $\alpha \geqq 0, \beta \geqq 0$ in the first operator. The other cases are similar. Let $\zeta \in C_{0}^{\infty}$, with $\zeta(x)=1$ in a neighborhood of $x=0$. We write

$$
\mu^{ \pm \alpha}=\left(\mu^{ \pm \alpha}\right)_{\zeta}+\left(\mu^{ \pm \alpha}\right)_{1-\zeta}
$$

where the subscript $\zeta$ or $1-\zeta$ replaces an operator with another whose kernel is multiplied by $\zeta(x-y)$ or $1-\zeta(x-y)$ respectively. Now $\left(\mu^{\alpha}\right)_{1-\zeta} e^{\beta|x|} \mu^{-\alpha} e^{-\beta|x|}$ is bounded by Propositions 7.1 and 7.2 and

$$
e^{\beta|x|}\left(\mu^{\alpha}\right)_{\zeta} \mu^{-\alpha} e^{-\beta|x|}=I+e^{\beta|x|}\left(\mu^{\alpha}\right)_{1-\zeta} \mu^{-\alpha} e^{-\beta|x|}
$$

is also bounded, for the same reason, as is $e^{\beta|x|} \mu^{-\alpha} e^{-\beta|x|}$. Thus our problem is reduced to bounding

$$
T=\left[\left(\mu^{\alpha}\right)_{\zeta}, e^{\beta|x|}\right] e^{-\beta|x|} .
$$


Because of the $\zeta$ in $T$, the unboundedness of $e^{\beta|x|}$ plays no role. In fact we choose $\zeta$ so that $\sum_{j} \zeta(\mathrm{x}+\mathrm{j})=1$. In momentum space, the kernel of

$$
T_{j}=\left[\left(\mu^{\alpha}\right)_{\zeta}, \zeta(x+j) e^{\beta|x|}\right] e^{-\beta j}
$$

is bounded by a multiple of

$$
f(p, q)=\left(\mu(p)^{\alpha}-\mu(q)^{\alpha}\right)(|p-q|+1)^{-2} .
$$

Since

$$
\sup _{p} \int|f(p, q)| d q<\infty
$$

$T_{j}$ is bounded. Moreover $\|T\| \leqq$ const $\sup _{j}\left\|T_{j}\right\|$ and the proof is complete.

Corollary 7.4. The space $A_{\delta}$ is the closure of $C_{0}^{\infty}(R)$ in the norm

$$
\|f\|_{\delta}=\left\|K_{\delta} f\right\|_{L_{2}}
$$

or in the norm

$$
\|f\|_{\delta}^{\prime}=\left\|K_{\delta}^{*} f\right\|_{L_{2}} .
$$

The norms $\|\cdot\|$ and $\|\cdot\|^{\prime}$ are equivalent.

Proof. We estimate

$$
\begin{aligned}
\|f\|_{\delta} & =\left\|K_{\delta} f\right\|=\left\|K_{\delta}\left(K_{\delta}^{*}\right)^{-1} K_{\delta}^{*} f\right\| \\
& \leqq\left\|K_{\delta}\left(K_{\delta}^{*}\right)^{-1}\right\|\|f\|_{\delta}^{\prime} \leqq\left\|K_{\delta}\left(K_{\delta}^{*}\right)^{-1}\right\|\left\|K_{\delta}^{*} K_{\delta}^{-1}\right\|\|f\|_{\delta} .
\end{aligned}
$$

However

$$
K_{\delta}\left(K_{\delta}^{*}\right)^{-1}=e^{\delta_{2}|x|} \mu^{\delta_{1}} e^{-\delta_{2}|x|} \mu^{-\delta_{1}},
$$

and

$$
K_{\delta}^{*} K_{\delta}^{-1}=\mu^{\delta_{1}} e^{\delta_{2}|x|} \mu^{-\delta_{1}} e^{-\delta_{2}|x|} .
$$

Applying the theorem to (7.12)-(7.13) shows that they are bounded, so $\|\cdot\|$ and $\|\cdot\|^{\prime}$ are equivalent.

Lemma 7.5. Let $\delta<1$ and let $v(x)$ be bounded, positive and square integrable. Let $T(x, y)$ be the kernel of an integral operator satisfying

$$
|T(x, y)| \leqq v(x) v(y)|x-y|^{-\delta} .
$$

Then $T$ is compact.

Proof. Let $\chi_{n}(x, y)$ be the characteristic function of $|x-y|>n^{-1}$, and let $T_{n}(x, y)$ $=T(x, y) \chi_{n}(x, y)$. Then $T_{n}$ is Hilbert Schmidt, and hence compact. Also

$$
\left\|T-T_{n}\right\| \leqq \sup _{x} \int_{|x-y|<n^{-1}} v(x) v(y)|x-y|^{-\delta} d y \leqq O\left(n^{-1+\delta}\right) .
$$

Hence $T$, as the norm limit of compact operators, is compact. 
Theorem 7.6. Let $\alpha<\beta, \gamma<\varepsilon$, with $\alpha ; \beta \in(-a, a), \gamma, \varepsilon \in(-1,1)$. Let

$T \equiv e^{+\alpha|x|} \mu^{+\gamma} e^{-\beta|x|} \mu^{-\varepsilon}$.

(i) $T$ is compact.

(ii) If $\varepsilon-\gamma>\frac{1}{2}, T$ is Hilbert Schmidt.

(iii) If $\varepsilon-\gamma>1, T$ is trace class.

Proof. We write

$$
T=\left(e^{\alpha|x|} \mu^{-(\varepsilon-\gamma)} e^{-\beta|x|}\right)\left(e^{\beta|x|} \mu^{\varepsilon} e^{-\beta|x|} \mu^{-\varepsilon}\right) .
$$

The second factor in (7.16) is bounded by Theorem 7.3. We now show that the first factor is compact, bounded or Hilbert Schmidt. Let $S$ denote this first factor. Then by (7.1), for any $\delta>0$, and assuming $0<\varepsilon-\gamma<1$,

$$
\begin{aligned}
|S(x, y)| & \leqq \text { const } e^{\alpha|x|}|x-y|^{-1+(\varepsilon-\gamma)} e^{-a|x-y|} e^{-\beta|y|} \\
& \leqq \text { const } e^{-(\beta-\alpha)|y|}|x-y|^{-1+(\varepsilon-\gamma)} e^{-(a-\alpha)|x-y|} \\
& \leqq \text { const } e^{-\delta|x|} e^{-(\beta-\alpha-\delta)|y|} e^{-(a-\alpha-\delta)|x-y|}|x-y|^{-1+\varepsilon-\gamma}
\end{aligned}
$$

By Lemma 7.5, $S$ is compact. Furthermore, if $\varepsilon-\gamma>\frac{1}{2}$, then $S$ is Hilbert Schmidt. If $\varepsilon-\gamma>1,0<\delta<\beta-\alpha$,

$$
S=\left(e^{\alpha|x|} \mu^{-(\varepsilon-\gamma) / 2} e^{-(\beta-\delta)|x|}\right)\left(e^{(\beta-\delta)|x|} \mu^{-(\varepsilon-\gamma) / 2} e^{-\beta|x|}\right) .
$$

Each factor in parentheses in (7.18) is Hilbert Schmidt, by (ii). Thus $S$, as the product of two Hilbert Schmidt operators, is trace class.

Proposition 7.7. Let $\delta_{2}>0$. The norm

$$
\|f\|_{\delta} \equiv \max _{ \pm}\left\|e^{ \pm \delta_{2} x} \mu^{\delta_{1}} f\right\|_{L_{2}}
$$

is equivalent to the norm $\|f\|_{\delta}$.

Proof. For $\alpha \geqq 0$, we have the pointwise bound

$$
\exp ( \pm \alpha x) \leqq \exp \alpha|x| \leqq \exp (\alpha x)+\exp (-\alpha x)
$$

Thus

$$
\|f\|_{\delta} \leqq\|f\|_{\delta} \leqq 2\|f\|_{\delta} .
$$

Proposition 7.8. Let $\left|\delta_{2}\right|<a$. Then as an operator from $A_{\delta}$ to $A_{\delta}$,

$$
\left\|\left|e^{-t \mu}\left\|\left.\right|_{A_{\delta}}=\sup _{f}\right\| e^{-t \mu} f\left\|\left.\right|_{\delta} /\right\|\right| f\right\|_{\delta} \leqq \exp \left(-t\left\{a^{2}-\delta_{2}^{2}\right\}^{1 / 2}\right) .
$$

Proof. First we note that the result for $\delta_{2}<0$ follows from the result for $\delta_{2} \geqq 0$. In fact if $T: A_{\delta} \rightarrow A_{\delta}$ is bounded with norm $\|T\|_{A_{\delta}}$, then $T^{*}: A_{\delta}^{*} \rightarrow A_{\delta}^{*}$ is bounded with the same norm. By (6.13), $A_{\delta}^{*}=A_{-\delta}$, so we take $\delta_{2} \geqq 0$; let $T=\exp (-t \mu)$. We show that $\|T\|_{A_{\delta}}$ is bounded as in (7.21). By Proposition 7.7, this shows that $T$ is bounded on $A_{\delta}$. For convenience, we choose $f$ in the dense set $C_{0}^{\infty}$ in $A_{\delta}$, so $f(p)$ is entire. Then

$$
\left\|e^{ \pm \alpha x} \mu^{\beta} T f\right\|_{L_{2}}=\left\|\mu(p \pm i \alpha)^{\beta} \exp (-t \mu(p \pm i \alpha)) \tilde{f}(p \pm i \alpha)\right\|_{L_{2}} .
$$


We note that

$$
\begin{aligned}
\operatorname{Re} \mu(p \pm i \alpha) & =\operatorname{Re}\left(p^{2}+a^{2}-\alpha^{2} \pm 2 i p \alpha\right)^{1 / 2} \\
& \geqq\left(p^{2}+a^{2}-\alpha^{2}\right)^{1 / 2} \geqq\left(a^{2}-\alpha^{2}\right)^{1 / 2} .
\end{aligned}
$$

Thus with $\alpha=\delta_{2}, \beta=\delta_{1}$,

$$
\begin{aligned}
\|\mid T f\|_{\delta} & \leqq \exp \left(-t\left(a^{2}-\alpha^{2}\right)^{1 / 2}\right) \max _{ \pm}\left\|\mu(p \pm i \alpha)^{\beta} \tilde{f}(p \pm i \alpha)\right\|_{L_{2}} \\
& =\exp \left(-t\left(a^{2}-\alpha^{2}\right)^{1 / 2}\right) \max _{ \pm}\left\|e^{ \pm \alpha x} \mu^{\beta} f\right\|_{L_{2}} \\
& =\exp \left(-t\left(a^{2}-\alpha^{2}\right)^{1 / 2}\right)\|f\|_{\delta} .
\end{aligned}
$$

Proposition 7.9. Let $T=\left(e^{-\mu} \otimes e^{-\mu}\right)$. Then acting on $A_{\delta}$ as functions of $p_{\text {rel }}$ with $p_{\text {tot }}$ fixed,

$$
\left\|T^{t} \mid\right\|_{A_{\delta}} \leqq \exp \left(-2 t \sqrt{a^{2}+p_{\text {tot }}^{2}-\delta_{2}^{2}}\right)
$$

Proof. As above, we assume $\delta_{2} \geqq 0$. Then

$$
\begin{aligned}
& \left\|e^{ \pm \alpha x_{\text {rel }}} \mu^{\beta} T f\right\|_{L_{2}} \\
& \quad=\left\|\mu\left(p_{\text {rel }} \pm i \alpha\right)^{\beta} \exp \left\{-t \mu\left(p_{\text {tot }}+p_{\text {rel }} \pm i \alpha\right)-t \mu\left(p_{\text {tot }}-p_{\text {rel }} \pm i \alpha\right)\right\} \tilde{f}\left(p_{\text {rel }} \pm i \alpha\right)\right\|_{L_{2}}
\end{aligned}
$$

using $p_{\text {tot }}=\frac{1}{2}\left(p_{1}+p_{2}\right), p_{\text {rel }}=\frac{1}{2}\left(p_{1}-p_{2}\right)$. Then as in (7.23),

$$
\sum_{ \pm} \operatorname{Re} \mu\left(p_{\text {tot }} \pm p_{\text {rel }} \pm i \alpha\right) \geqq 2\left(a^{2}+p_{\text {tot }}^{2}-\alpha^{2}\right)^{1 /}
$$

Thus

$$
\left\|e^{ \pm \alpha x_{\mathrm{rel}}} \mu^{\beta} T f\right\|_{L_{2}} \leqq e^{-2 t\left(a^{2}+p_{\mathrm{tot}}^{2}-\alpha^{2}\right) 1 / 2}\left\|\mu\left(p_{\mathrm{rel}} \pm i \alpha\right)^{\beta} \tilde{f}\right\|_{L_{2}} .
$$

Corollary 7.10. For $\operatorname{Re} E<2\left(a^{2}+p_{\text {tot }}^{2}-\delta_{2}^{2}\right)^{1 / 2}$,

$$
\left.\left\|\left(1-e^{E} e^{-\mu} \otimes e^{-\mu}\right)^{-1}\right\|\right|_{A_{\delta} \rightarrow A_{\delta}} \leqq\left(1-e^{-\left[2\left(a^{2}+p_{\mathrm{tot}}^{2}-\delta_{2}^{2}\right)^{1 / 2}-\operatorname{Re} E\right]}\right)^{-1}
$$

Remark. With $T$ as in Proposition 7.9, the Corollary bounds

$$
\tilde{T}(E) \equiv \sum_{j \geqq 0} T^{j} e^{j E}
$$

We now show that $\tilde{T}(E)$ has a two sheeted analytic continuation around a square root branch point at threshold. For simplicity, we take $p_{\text {tot }}=0$ and we do not analyze the singularity at threshold.

\section{Proposition 7.11. For}

$$
\zeta=\left(4 a^{2}-E^{2}\right)^{1 / 2} \neq 0,
$$

the operator

$$
\tilde{T}(E(\zeta))=\frac{1}{1-\exp \left[\left(4 a^{2}-\zeta^{2}\right)^{1 / 2}-\left(4 a^{2}+4 p_{\text {rel }}^{2}\right)^{1 / 2}\right]}
$$

acting from $A_{\left(\delta_{1}, \delta_{2}\right)}$ to $A_{\left(\delta_{1},-\delta_{2}\right)}, \delta_{2}>0$, has a bounded analytic continuation in $\zeta$ from $\operatorname{Re} \zeta>2 \delta_{2}$ (i.e. $\operatorname{Re} E<2\left(a^{2}-\delta_{2}^{2}\right)^{1 / 2}$ ) to the region $\operatorname{Re} \zeta>-2 \delta_{2}$. 
Proof. We see that $\tilde{T}(E(\zeta))$ has poles in $p_{\text {rel }}$ at

$$
p_{\text {rel }}= \pm i \zeta / 2
$$

As long as the poles do not cross the strip $\left|\operatorname{Im} p_{\text {rel }}\right| \leqq \delta_{2}$, i.e. as long as $\operatorname{Re} \zeta>-2 \delta_{2}$, the contour integral defining the inner product

$$
\left\langle f, \tilde{T}(E) f>_{L_{2}}, \quad f \in A_{\delta},\right.
$$

as written in the momentum space variable $p_{\text {rel }}$, can be deformed to avoid the poles (7.29). This procedure defines the analytic continuation of (7.28) to $\operatorname{Re} \zeta>-2 \delta_{2}$. Mapping back to the variable $E$ gives the two sheeted analytic continuation of (7.27) around the square root branch point $E=2 a$. For $\zeta \neq 0$, the contour can be chosen so that (7.28) is bounded along the contours. As $\zeta \rightarrow 0$, the poles come together, and the contour, which must go above one pole and below the other, cannot be bounded away from the poles. See also $[3,4,12]$.

It is only in the analytic continuation argument that we use the fact that the functions $f \in A_{\delta}$ are symmetric in $x$-space. Because of this assumption, (7.30) can be written in momentum space as

$$
\langle f, \tilde{T}(E) f\rangle_{L_{2}}=\int_{-\infty}^{\infty} \tilde{\tilde{f}}(p)(\tilde{T}(E) \tilde{f})(p) d p .
$$

Thus no absolute values occur in (7.31) and the analytic continuation in $E$ can be accompanied by the deformation of the $p$ integration contour (inside the strip $\left.|\operatorname{Im} p|<\delta_{2}\right)$ to avoid the poles (7.29) of $\tilde{T}(E(\zeta))$. By the above argument, if $C(\zeta)$ denotes the deformed contour,

$$
\left|\langle f, \tilde{T}(E) f\rangle_{L_{2}}\right| \leqq \sup _{C(\zeta)}|\tilde{T}(E(\zeta))| \int_{C(\zeta)}|\tilde{\bar{f}}(p) \tilde{f}(p)| d p .
$$

since the sup is dominated by $O(1)$. For $|\operatorname{Im} p|$ bounded away from $\delta_{2}$, $|\tilde{f}(p)| \leqq$ const $\|f\|_{A_{\delta}}$. Thus in (7.31),

$$
\left|\int_{-L}^{L} \tilde{\tilde{f}}(p) \tilde{f}(p) \tilde{T}(E) d p\right| \leqq \mathrm{const}\|f\|_{A_{\delta}}^{2} .
$$

The tails, $\int_{-\infty}^{-L}+\int_{L}^{\infty}$ are bounded directly, and can be continued analytically to $\operatorname{Re} E \leqq L$ without deformation of contour, as in Proposition 7.9, and Corollary 7.10. Since $L$ is arbitrary, we get the desired analytic continuation.

\section{One Particle Lines in a Two Particle Channel}

We modify the two particle cluster expansion [8] applied to $S_{0, T}$ in order to define our integral equation. The first modification is inclusion of virtual contraction terms to the : $\phi(T)^{2}$ : observable, to allow resummation of one particle lines, as explained in $\S 5$. The second modification, which is new to this section, is the inclusion of virtual interaction terms between the independent one particle lines. 
The operator

$$
e^{-t \mu} \otimes e^{-t \mu}
$$

generates dynamics in two independent one particle lines, and we want to find this operator by resumming certain terms from the expansion of : $\phi\left(x_{1}\right) \phi\left(x_{2}\right):$. It is easy to find a candidate for this resummation, namely all terms in which there is no contraction between $x_{1}$ and $x_{2}$ vertices, nor any overlap between the $x_{1}$ and $x_{2}$ contours. Each term of this nature factors, and thus expresses some type of independent dynamics for $x_{1}$ and $x_{2}$. However, the sum of these terms does not factor, and does not define independent dynamics for $x_{1}$ and $x_{2}$. In fact, some terms, expected as part of the $e^{-t \mu}$ dynamics for $x_{1}$ do not occur, because the region of space required to insert this term in the expansion is already occupied by other vertices and/or contours belonging to $x_{2}$ or to $x_{1}$ and $x_{2}$ jointly. To compensate, we add and subtract these missing terms. With the addition, the sum of the independent terms also factors, and can be identified with (8.1). When subtracted, the terms are called virtual interaction terms; they contribute along with the direct interaction terms to the Bethe-Salpeter kernel.

In more detail, we restrict the momenta of each $x_{j}$ test function as in (5.7). Using a smooth momentum space cutoff function, we write the $x_{j}$ test function as a sum of two test functions. The first has high momentum and the second has purely low momentum. The term with the high momentum has an enhanced decay rate. This fact can be obtained from the convergence proof of [8]. Because of the high decay rate ( $\geqq 2 m_{0}-\varepsilon$ for the single particle) a simplier expansion, not requiring a backward movement in time is possible for the high momentum term. This expansion will be explained later.

The more difficult term involves low momentum. We now explain the expansion for these terms. Let $: \phi^{2}:_{W}$ denote normal ordering relative to the bare vacuum. This is the normal ordering used in [8]. By adding and subtracting a constant term and a term linear in $\phi$, we can pass from $: \phi^{2}:$ to $: \phi^{2}:_{W}$ or back, as in (2.13). We use this identity to start the expansion with $: \phi^{2}{ }_{W}$, but to yield $: \phi^{2}$ : terms $(1<t=i \leqq T)$ as a result of the expansion. It is also used to write the time $T: \phi^{2}$ : observable as : $\phi^{2}:_{W}+\phi+$ const. The linear term $\left.\phi\right|_{t=0}$ is expanded as in $\S 5$, as will be explained in more detail below.

The $: \phi^{2}:\left._{W}\right|_{t=0}$ is expanded according to the 2-particle expansion of [8]. Virtual terms are added and subtracted, for interaction with the time $T$ observables and also for interaction between the two one particle lines. For terms without interaction between the two $\phi^{2}$ lines, each $\phi$ is returned independently to $t=1$. The expansion terminates with any of these terms:

(i) a constant (i.e. $\phi$-independent) term,

(ii) a term linear in $\phi$, returned to $t=1$,

(iii) two independent $\phi$ 's in the form : $\phi\left(y_{1}\right) \phi\left(y_{2}\right)$ :, each returned to $t=1$,

(iv) a : $\phi^{2}$ : at integer time $t=i, 1 \leqq i<T$ with direct or virtual interaction between the two variables $y_{1}$ and $y_{2}$

(v) direct or virtual contraction to $: \phi^{2}:(T)$, the time $T$ observable.

Because of the virtual terms in (v), the terms (i) and (ii) are $T$ independent; because of normal ordering in $: \phi^{2}:$, they sum to zero. The terms (iii) are exactly the tensor product of the one particle line expansion of $\S 5$, and thus can be 
identified with the dynamics (8.1). These terms are then $e^{-\mu} \otimes e^{-\mu} S_{1, T}$. The terms (iv) are proportional to $S_{i, T}$, and give $B_{0, i}^{\text {amp }} S_{i, T}$, where $B_{0, i}^{\text {amp }}$ denotes the sum of the proportionality operators for fixed $i$. Finally the terms $(v)$ have three particle decay, and are bounded by $O\left(\exp \left(-3\left(m_{0}-\varepsilon\right) T\right)\right)$. We denote the terms $(\mathrm{v})$ by $A_{0, T}^{\mathrm{amp}}$.

The expansion of $\left.\phi\right|_{t=0}$ follows $\S 5$. All terms linear in $\phi$ are returned to $t=1$. The expansion terminates with terms of type (i), (ii), (iv) or (v), but gives no contribution to the (iii) terms.

In the high momentum term, the two $\phi$ 's in $: \phi^{2}:_{W}$ are moved forward in time independently until they interact with each other (direct or virtual interaction or until they contract (direct or virtual contraction) to a time $T$ observable. This produces terms of the type (i), (ii), (iv), (v), as above, but not of type (iii). The (i) and (ii) terms sum to zero, and the (iv) and (v) terms are estimated as below. We note that as long as the two lines do not interact, momentum is conserved within each line. In particular, the high momentum property is retained until interaction.

In both high and low momentum terms, we require an independent average over the lattice displacement $\theta \in(0,1]$ for each of the independent, one particle lines. This means that the contours for the distinct lines are inserted along distinct, incompatible lattices.

The estimates on the (iv) and (v) terms of the expansion follow from [8], because all terms, including virtual terms, arise from a finite number of sums, differences or products of convergent expansions. Thus the (iv) and (v) terms are absolutely convergent in the norms of [8]. In view of regularization (see $\S 9,10$ ), they are also bounded and absolutely convergent in the operator norms on $A_{\delta}$, on the space of equal time functions.

In summary, we have

$$
S_{0, T}=e^{-\mu} \otimes e^{-\mu} S_{1, T}+\sum_{i=1} B_{0, i}^{\mathrm{amp}} S_{i T}+A_{0, T}^{\mathrm{amp}} .
$$

Iterating the expansion, we obtain (1.6) with

$$
\begin{aligned}
& A_{0, T}=\sum_{0 \leqq j \leqq T}\left(e^{-\mu} \otimes e^{-\mu}\right)^{j} A_{j, T}^{\mathrm{amp}} \\
& B_{0, T}=\sum_{0 \leqq j \leqq T-1}\left(e^{-\mu} \otimes e^{-\mu}\right)^{j} B_{j, T}^{\mathrm{amp}} .
\end{aligned}
$$

Thus

$$
\begin{aligned}
& \tilde{A}(E)=\sum_{0 \leqq T} A_{0, T} e^{E T}=\left(I-e^{E} e^{-\mu} \otimes e^{-\mu}\right)^{-1} \sum_{0 \leqq T} A_{0, T}^{\mathrm{amp}} e^{E T}=\tilde{T}(E) \tilde{A}^{\mathrm{amp}}(E), \\
& \tilde{B}(E)=\sum_{0 \leqq T} B_{0, T} e^{E T}=\left(I-e^{E} e^{-\mu} \otimes e^{-\mu}\right)^{-1} \sum_{1<T} B_{0, T}^{\mathrm{amp}} e^{E T}=\tilde{T}(E) \tilde{B}^{\mathrm{amp}}(E) .
\end{aligned}
$$

Thus we write (1.6) as

$$
\tilde{S}(E)=\tilde{A}(E)+\tilde{B}(E) \tilde{S}(E) .
$$

\section{Decay Estimates in $x$-Spaces}

The two particle expansion of $\S 8$ introduces seven operators which map kernels of const, $\phi(x)$ or : $\phi\left(x_{1}\right) \phi\left(x_{2}\right)$ : into kernels of const, $\phi$ or : $\phi^{2}$ : at possibly later times. 
The simplest of these operators is $e^{-\mu} \otimes e^{-\mu}$. This operator is described in $\S 7$, and it acts on the kernel of $: \phi^{2}$ : in the mapping

$$
: \phi\left(x_{1}\right) \phi\left(x_{2}\right):\left.\right|_{t=0} \rightarrow: \phi\left(y_{1}\right) \phi\left(y_{2}\right):\left.\right|_{t=1} .
$$

Another simple operation is re-Wick ordering $\delta \alpha_{0}$. Since integration by parts yields

$$
\delta \alpha_{0} \in A_{\frac{3}{2}-\varepsilon, m-\varepsilon}=A_{-\frac{3}{2}+\varepsilon,-m+\varepsilon}^{*}
$$

$\delta \alpha_{0}$ is a bounded linear functional on all $A_{\delta}$ spaces we consider. Also $\tilde{\alpha}_{1}\left(p, x_{\text {rel }}\right)$ for fixed $p$ satisfies

$$
\alpha_{1}(p, \cdot) \in A_{\frac{3}{2}-\varepsilon, \delta_{2}}=A_{-\frac{3}{2}+\varepsilon,-\delta_{2}}^{*}, \quad \delta_{2}>0 .
$$

and thus is bounded on all $A_{\delta}$ spaces we consider.

Let $\mathscr{K}_{j}^{\text {(iv) }}$ denote the operator which is the sum of all (iv) terms associated with the map

$$
: \phi\left(x_{1}\right) \phi\left(x_{2}\right):\left._{W}\right|_{t=0} \rightarrow: \phi\left(y_{1}\right) \phi\left(y_{2}\right):\left.\right|_{t=j} .
$$

The operator $\mathscr{K}^{(\mathrm{iv})}$ has a kernel depending on $x_{\text {rel }}, y_{\text {rel }}, x_{\text {tot }}-y_{\text {tot }}$.

We similarly define an operator $\mathscr{K}_{T}^{(\mathrm{v})}$ by the sum of type (v) terms. Then $\mathscr{K}_{T}^{(\mathrm{v})}$ is determined as a bilinear form on the kernels $f\left(x_{\text {rel }}, p_{\text {rel }}\right)$ and $g\left(y_{\text {rel }}, p_{\text {rel }}\right)$ of the time $t=0$ and time $t=T$ observables.

Namely at time $t=0$, the observable is

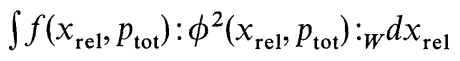

and at Euclidean time $t=T$, it is

$$
\begin{aligned}
& \int g\left(y_{\text {rel }}, p_{\text {tot }}\right): \phi^{2}\left(y_{\text {rel }}, p_{\text {tot }}\right): d y_{\text {rel }} \\
& =\int g\left(y_{\text {rel }}, p_{\text {tot }}\right): \phi^{2}\left(y_{\text {rel }}, p_{\text {tot }}\right):{ }_{W} d y_{\text {rel }} \\
& \quad-\int g\left(y_{\text {rel }}, p_{\text {tot }}\right) \tilde{\alpha}_{1}\left(p_{\text {tot }}, y_{\text {rel }}\right) d y_{\text {rel }} \tilde{\phi}\left(p_{\text {tot }}\right) \\
& \quad+\int g\left(y_{\text {rel }}, p_{\text {tot }}\right) \delta \alpha_{0}\left(y_{\text {rel }}\right) d y_{\text {rel }} .
\end{aligned}
$$

With $f \in A_{\delta}$, as a function of $x_{\text {rel }}, g \in A_{\delta^{1}}$, then $\mathscr{K}_{T}^{(\mathrm{v})}$ is an operator from $A_{\delta}$ to $A_{\delta^{1}}^{*}=A_{-\delta^{1}}$.

Also let $\mathscr{L}_{j}^{\text {(iv) }}$ and $\mathscr{L}_{j}^{(\mathrm{v})}$ be associated with the map

$$
\left.\phi(x)\right|_{t=0} \rightarrow: \phi\left(y_{1}\right) \phi\left(y_{2}\right):\left.\right|_{t=j}
$$

and with $\phi(x)$ contractions at time $T$. Then $\mathscr{L}^{(\text {iv) }}$ has a kernel depending on $y_{\text {rel }}$, $x-y_{\text {tot }}$.

For comparison with [8], we restrict $\mathscr{K}, \mathscr{L}$ to functions supported in products of unit intervals. The $\mathscr{K}, \mathscr{L}$ are bounded from $L_{2}$ to $L_{2}$. According to $\S 6$, the unit lattice squares in $x_{1}, x_{2}$ space and in $y_{1}, y_{2}$ space can be rotated by $\pi / 4$ so that they are alligned with $x_{\text {rel }}, x_{\text {tot }}, y_{\text {rel }}, y_{\text {tot }}$. Let $\Delta_{x}$, etc. denote the characteristic function of such a square. A minor improvement in the result of [8] includes some $x$ as well as 
$t$ exponential decay. With $: \phi^{2}:$ as the time $T$ observable this yields

$$
\begin{aligned}
\left\|\Delta_{x} \mathscr{K}_{T}^{(\mathrm{v})} \Delta_{y}\right\|_{L_{2} \rightarrow L_{2}^{*}} & \leqq e^{-3(m-\varepsilon) T} e^{-\varepsilon^{\prime}\left|x_{\mathrm{tot}}-y_{\mathrm{tot}}\right|} \\
& \leqq\left[e^{-\varepsilon^{\prime}\left|x_{\mathrm{rel}}-y_{\mathrm{rel} 1}\right|}+e^{-\varepsilon^{\prime}\left|x_{\mathrm{re} 1}+y_{\mathrm{rel}}\right|}\right] .
\end{aligned}
$$

The type (iv) terms have an improved decay because the interaction between the two one particle lines give graphs which are connected, and hence decay in $\left|x_{\text {rel }}\right|+\left|y_{\text {rel }}\right|$. Thus

$$
\left\|\Delta_{x} \mathscr{K}_{j}^{(\mathrm{iv})} \Delta_{j}\right\|_{L_{2} \rightarrow L_{2}} \leqq e^{-3(m-\varepsilon) j} e^{-\varepsilon^{\prime}\left|x_{\mathrm{tot}}-y_{\mathrm{tot}}\right|} e^{-\varepsilon^{\prime}\left|x_{\mathrm{re} 1}\right|-\varepsilon^{\prime}\left|y_{\mathrm{re} 1}\right|} .
$$

The graphs in $\mathscr{L}$ are all connected.

$$
\left\|\Delta_{x} \mathscr{L}_{j}^{\text {(iv) }} \Delta_{y}\right\|_{L_{2} \rightarrow L_{2}} \leqq e^{-3(m-\varepsilon) j} e^{-\varepsilon^{\prime}\left|x-y_{\text {tot }}\right|} e^{-\varepsilon^{\prime}\left|y_{\mathrm{re} 1}\right|}
$$

and

$$
\left\|\Delta_{x} \mathscr{L}_{j}^{(\mathrm{v})} \Delta_{j}\right\|_{L_{2} \rightarrow L_{2}^{*}} \leqq e^{-3(m-\varepsilon) T} e^{-\varepsilon^{\prime}\left|x-y_{\mathrm{tot}}\right|} e^{-\varepsilon^{\prime}\left|y_{\mathrm{re} 1}\right|} .
$$

Next we consider the operators $\mathscr{K}, \mathscr{L}$ at fixed total momentum $p$, as mappings on the function of relative variables:

$$
\begin{aligned}
& \mathscr{K}_{j}^{(\mathrm{iv})}: A_{\delta}^{*}=A_{-\delta} \rightarrow A_{\delta}, \quad \mathscr{K}_{T}^{(\mathrm{v})}: A_{\delta} \rightarrow A_{\delta} \\
& \mathscr{L}_{j}^{\text {(iv) }} \in A_{\delta}, \quad \mathscr{L}_{T}^{(\mathrm{v})} \in A_{\delta} .
\end{aligned}
$$

For $\delta_{1}=0$ these four norms are bounded as follows:

Theorem 9.1. For $\delta_{2}$ sufficiently small and greater than zero, the four operators (9.11) are bounded. The norms are $O\left(e^{-3(m-\varepsilon) j}\right)$ for $\mathscr{K}_{j}$ and $\mathscr{L}_{j}$. Moreover $\mathscr{K}$ and $\mathscr{L}$ are complex analytic in $p_{\text {tot }}$, for $\left|\operatorname{Im} p_{\text {tot }}\right|$ small.

Proof. For $\mathscr{K}_{j}^{\text {(iv) }}$ we have

$$
\begin{aligned}
\left\|\mathscr{K}_{j}^{(\mathrm{iv})}\right\|_{A_{\delta} \rightarrow A_{\delta}} & \leqq \sum e^{\delta_{2}\left|x_{\mathrm{re} 1}\right|+\delta_{2}\left|y_{\mathrm{rel} 1}\right|}\left\|\Delta_{x} \mathscr{K}_{j}^{(\mathrm{iv})} \Delta_{y}\right\|_{L_{2} \rightarrow L_{2}} \\
& \leqq O\left(\sum e^{-3(m-3) j-\left|x_{\mathrm{tot}}-y_{\mathrm{tot}}\right|\left(\varepsilon^{\prime}-\left|\operatorname{Im} p_{\mathrm{tot}}\right|\right)} \leqq O\left(e^{-3(m-\varepsilon) j}\right) .\right.
\end{aligned}
$$

Similarly

$$
\left\|\mathscr{K}_{T}^{(\mathrm{v})}\right\|_{A_{\delta} \rightarrow A_{\delta}} \leqq \sum e^{-\delta_{2}\left|x_{\mathrm{re} 1}\right|+\delta_{2}\left|y_{\mathrm{re} 1}\right|}\left\|\Delta_{x} \mathscr{K}_{T}^{(\mathrm{v})} \Delta_{y}\right\|_{L_{2} \rightarrow L_{2}^{*}} \leqq O\left(e^{-3(m-\varepsilon) T}\right) .
$$

The remaining bounds on

$$
\begin{gathered}
\left\|\mathscr{L}_{j}^{\text {(iv) }}\right\|_{A_{\delta}} \leqq \sum e^{\delta\left|y_{\mathrm{rel} 1}\right|}\left\|\Delta_{x} \mathscr{L}_{j}^{\text {(iv) }} \Delta_{y}\right\|_{L_{2} \rightarrow L_{2}} \leqq e^{-3(m-\varepsilon) j} \\
\left\|\mathscr{L}_{T}^{(\mathrm{v})}\right\|_{A_{\delta}} \leqq \sum e^{\delta\left|y_{\mathrm{re} 1}\right|}\left\|\Delta_{x} \mathscr{L}_{T}^{(\mathrm{v})} \Delta_{y}\right\|_{L_{2} \rightarrow L_{2}^{*}} \leqq e^{-3(m-\varepsilon) T}
\end{gathered}
$$

are similar, to complete the proof.

The proof of (9.12) applies to the error terms in (5.17) and shows their contribution to $\tilde{\alpha}_{1}$ lies in $A_{0, \delta_{2}}, \delta_{2}>0$.

\section{Smoothing and Compactness in $x$-Space}

In this section we exhibit the smoothing property of the operators $\mathscr{K}$ and $\mathscr{L}$ defined in $\S 8$ and $\S 9$. In particular, this gives the properties of $A^{\text {amp }}$ and $B^{\text {amp }}$ on 
the spaces $A_{\delta}$. Our main technical result, which leads to an analysis of $S_{0, T}$ is the following:

Theorem 10.1. The connected expansion terms ( $\$ 8$, type iv) define bounded operators $B_{0, j}^{\mathrm{amp}}$ such that

$$
B_{0, j}^{\mathrm{amp}}: A_{-\delta} \rightarrow A_{\delta}
$$

with

$$
\left\|B_{0, j}^{\mathrm{amp}}\right\|_{A_{-\delta} \rightarrow A_{\delta}} \leqq O(\lambda) e^{-3(m-\varepsilon) j}
$$

when

$$
\delta_{1} \leqq 2-\varepsilon, \quad \delta_{2}<\varepsilon,
$$

for some $\varepsilon>0$ sufficiently small. The terms of $\S 8$, type $\mathrm{v}$ define the bounded operator $A_{0, T}^{\mathrm{amp}}$, where

$$
A_{0, T}^{\mathrm{amp}}: A_{-1+\varepsilon, \delta_{2}} \rightarrow A_{1-\varepsilon, \delta_{2}}
$$

with

$$
\left\|A_{0, T}^{\mathrm{amp}}\right\| \leqq O(1) e^{-3(m-\varepsilon) T} .
$$

Moreover, $B_{0, j}^{\mathrm{amp}}$ is compact on the indicated spaces, and trace class if $\delta_{1} \leqq \frac{3}{2}-\varepsilon$.

Remark. It follows that the Laplace transformed operators

$$
\begin{aligned}
& A^{\mathrm{amp}}(E)=\sum_{0 \leqq T} A_{0, T}^{\mathrm{amp}} e^{E T} \\
& B^{\mathrm{amp}}(E)=\sum_{0 \leqq T} B_{0, T}^{\mathrm{amp}} e^{E T}
\end{aligned}
$$

are complex analytic in $E$ for $\operatorname{Re} E \leqq 3\left(m_{0}-\varepsilon\right)$. This statement is true for $\tilde{A}^{\text {amp }}$ and $\tilde{B}^{\text {amp }}$ as operators on functions of the relative position variable. In particular, they are bounded as operators from $A_{\delta}$ to $A_{\delta^{\prime}}$ where $\delta, \delta^{\prime}$ are given by Theorem 10.1, and $\tilde{B}^{\mathrm{amp}}$ is compact, and has small norm as $\lambda \rightarrow 0$.

Proof. The improvement of Theorem 10.1 over the $\S 9$ involves a careful examination of the initial (and final) expansion steps. Each term at the end of the expansion has the structure given in $\S 8$. The sum of all expansion terms converge in the norms of [8]. We thus inspect individual terms. The $\mathscr{K}_{j}^{\text {(iv) }}$ terms, for example, have the structure

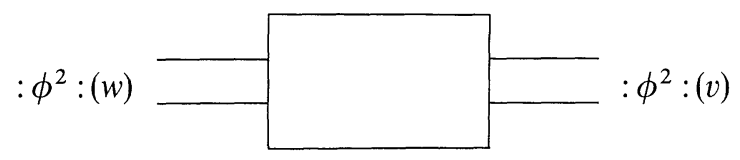

where $w$ is the kernel of $: \phi^{2}:(t=0)$ and $v$ is the kernel of $: \phi^{2}:(t=j)$. The initial expansion step inserts a contour around the $: \phi^{2}:(t=0)$ vertices; this contour may contain other vertices and partial contours. Likewise the expansion ends by 
removing the : $\phi^{l}:(t<j)$ vertices by a contour. In [8] estimates on the inside vertices $R(\phi)$ and function space integration was obtained by Schwarz inequality in path space

$$
\left|\int_{\text {inside }} e^{-P(\phi)} R(\phi) d \phi_{\text {inside }}\right| \leqq \mid\left(\int R^{2} d \phi \int e^{-2 P(\phi)} d \phi\right) .
$$

However, optimal bounds on the Schwinger functions, e.g. $\int e^{-P(\phi)} R(\phi) d \phi_{\text {inside }}$ improve on this estimate, see [5]. These optimal bounds are obtained by integration by parts of each field in $R(\phi)$, before applying the Schwarz inequality.

In expressions such as for $\mathscr{K}^{\text {(iv) }}$ in (10.7), this integration by parts produces "initial" and "final" vertices which contract with the $: \phi^{2}:(w)$ or $: \phi^{2}:(v)$. An analysis of these diagrams shows that their continuity in $v$ and $w$ lies not only in $L_{2}$, but in the appropriate Sobolev space for Theorem 10.1. For example, the dominant diagrams in $\mathscr{K}^{\text {(iv) }}$ have the form

$w$

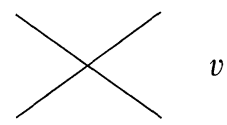

with a single $\phi^{4}$ vertex. Their momentum space decay is estimated for fixed $p_{\text {tot }}$ by $O(\lambda)\left\|\mu^{-2} w\right\|_{L_{2}}\left\|\mu^{-2} v\right\|_{L_{2}}$ in the variable $p_{\text {rel }}$. Here each line carries a factor $\mu^{-1}$, and the integration over the time of the intermediate vertex gives an additional factor $\mu^{-1}$ which can be divided between $w$ and $v$. More generally, a large $\mathscr{K}^{\text {(iv) }}$ diagram can be estimated by isolating a vertex on either end, and using and $L_{2}$ operator norm on the intermediate diagram:

$w$

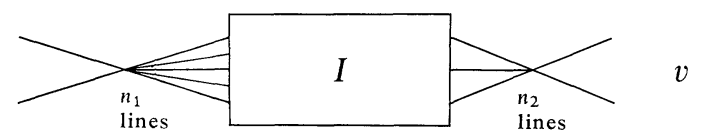

Then $I$ as an operator from $L_{2}\left(R^{n_{2}}\right)$ to $L_{2}\left(R^{n_{1}}\right)$ has an operator norm $\|I\|_{n_{1}, n_{2}}$ which can be estimated by the cluster expansion and Schwarz inequality as mentioned above. The single vertex diagrams have Hilbert-Schmidt norms $\leqq O(1)\left\|\mu^{-2} w\right\|\left\|\mu^{-2} v\right\|$ as above. The reduction to these norms is systematized in $[6, \S 6]$.

Other typical terms which arise involve $\alpha_{1}$. (The analysis of such terms completes the proof of Theorem 5.1.) These terms will have a dominant diagram

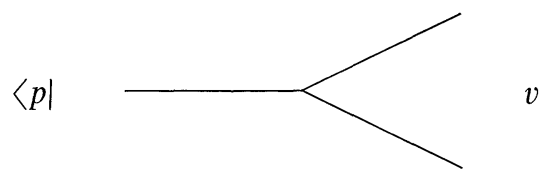


which as above is estimated by

$O(1)\left\|\mu^{-2} v\right\|$.

The dominant diagram for $\tilde{A}^{\text {amp }}(E)$ is

$w \longleftarrow v$

arising from the $T=0$ contribution. Here we obtain the estimate $O(1)\left\|\mu^{-1} v\right\|_{L_{2}}\left\|\mu^{-1} w\right\|_{L_{2}}$ arising from a factor $\mu^{-1}$ on each line.

We now return to the Eqs. (1.6), (8.3)-(8.7), (10.6)-(10.7). In Laplace transform space the solution for $\tilde{S}(E)$ is

$$
\tilde{S}(E)=[1-\tilde{B}(E)]^{-1} \tilde{A}(E) \text {. }
$$

To analyze bound states, it is sufficient to regard $\tilde{S}(E)$ as a bilinear form between a dense set of states. We use states $\theta_{p}=\int f\left(x_{\text {rel }}, p_{\text {tot }}\right): \phi\left(x_{1}\right) \phi\left(x_{2}\right): d x_{\text {rel }} \Omega$, dense in $\mathscr{H}(p)$ by $\S 3$. Thus we study the bilinear form $\langle g, \tilde{S}(E) f\rangle$, or equivalently, we realize (10.9) as an operator equation from $A_{-1+\varepsilon, \delta_{2}}$ to $A_{1-\varepsilon, \delta_{2}}=A_{-1+\varepsilon, \delta_{2}}^{*}$.

By Proposition 7.11 and Theorem 10.1,

$$
\tilde{A}(E): A_{-1+\varepsilon, \delta_{2}} \rightarrow A_{1-\varepsilon,-\delta_{2}},
$$

for $\operatorname{Re} E \leqq 3\left(m_{0}-\varepsilon\right)$, with an analytic continuation onto the second sheet, for $E$ not at threshold. Similarly

$$
\tilde{B}(E): A_{-2+\varepsilon,-\delta_{2}} \rightarrow A_{2-\varepsilon,-\delta_{2}}
$$

is bounded, and

$$
\tilde{B}(E): A_{-\frac{3}{2}+\varepsilon,-\delta_{2}} \rightarrow A_{\frac{3}{2}-\varepsilon,-\delta_{2}}
$$

is trace class, with norm (away from threshold)

$$
\|\tilde{B}(E)\|_{A_{\delta} \rightarrow A_{\delta}} \leqq O(\lambda)<1, \quad \text { for } \quad \delta_{1}>0, \quad \delta_{2}<0 .
$$

Thus $(I-\tilde{B}(E))^{-1}$ is bounded on the same spaces, and

$$
\tilde{S}(E): A_{-1+\varepsilon, \delta_{2}} \rightarrow A_{1-\varepsilon,-\delta_{2}}
$$

is bounded.

Near threshold, since $\tilde{B}(E)$ is compact, $(I-\tilde{B}(E))^{-1}$ and $\tilde{S}(E)$ are meromorphic, and the only possible point of accumulation of poles is at threshold.

The asserted properties of bound states follow. See $[3,4,12]$ for a more complete analysis of a similar situation.

\section{References}

1. Bros, J., Lasalle, M.: Analyticity properties and many body structure in general quantum field theory. III. Commun. Math. Phys. 54, 33-62 (1977)

2. Combescure, M., Dunlop, F.: $N$-particle irreducible functions in Euclidean quantum field theory. Ann. Phys. (N.Y.) to appear 
3. Dimock, J., Eckmann, J.P.: On the bound state in weakly coupled $\lambda\left(\phi^{6}-\phi^{4}\right)_{2}$. Commun. Math. Phys. 51, 41-54 (1976)

4. Dimock, J., Eckmann, J.P.: Spectral properties and bound state scattering for weakly coupled $P(\phi)_{2}$ models. Ann. Phys. 103, 289-314 (1977)

5. Glimm, J., Jaffe, A.: Two and three body equations. Commun. Math. Phys. 44, 293-320 (1975)

6. Glimm, J., Jaffe, A.: Positivity of the $\phi_{3}^{4}$ Hamiltonian. Fortschr. Physik 21, 327-376 (1973)

7. Glimm, J., Jaffe, A. : In: International symposium on mathematical problems in theoretical physics, Araki, H. (ed.). Lecture notes in physics, Vol. 39. Berlin, Heidelberg, New York: Springer 1975

8. Glimm, J., Jaffe, A., Spencer, T.: The Wightman axioms and particle structure in the $P(\phi)_{2}$ quantum field model. Ann. Math. 100, 585-632 (1974)

9. Koch, H.: Private communication

10. Gelfand, I., Shilov, G.: Generalized functions, Vol. II, III. New York: Academic Press 1967

11. Spencer, T.: The decay of the Bethe-Salpeter kernel in $P(\phi)_{2}$ quantum field. Commun. Math. Phys. 44, 143-164 (1975)

12. Spencer, T., Zirilli, F.: Scattering states and bound states in $\lambda P(\phi)_{2}$. Commun. Math. Phys. 49, 1-16 (1976)

13. Symanzik, K.: On the many particle structure of Green's functions in quantum field theory. J. Math. Phys. 1, 249-273 (1960)

Communicated by A. Jaffe

Received January 9, 1979 
